\title{
STIEFEL-WHITNEY HOMOLOGY CLASSES AND BORDISM
}

BY

\section{ETHAN AKIN}

ABSTRACT. We develop the theory of mod 2 Stiefel-Whitney homology classes for Euler polyhedra. We then describe a simple method of obtaining p.1. bordism theories. Finally, we define the ungraded bordism theory of Euler spaces and show that it is isomorphic to ordinary total homology.

In this paper we develop the theory of Stiefel-Whitney homology classes for a class of polyhedra more general than manifolds, christened Euler spaces by Halperin and Toledo [H-T].

Sullivan and I worked out the details of the first section after he returned from the Georgia conference where Cheeger presented a proof [Ch] of Whitney's unpublished result that the Stiefel-Whitney cohomology classes are dual to the skeleta of the derived subdivision of a triangulation of the manifold. Sullivan has since applied some of this work to analytic spaces [S]. Halperin and Toledo have generalized this work in several directions. They have shown that these classes are integral in certain cases and have extended the duality theorem to the noncompact case, too. Their argument, in contrast to that of Cheeger, is purely combinatorial. For simplicity, we will deal for the most part with only the mod 2 compact case.

The second part of the paper describes a simple method of obtaining p.l. bordism theories and computes some examples.

In the third section we define the ungraded bordism theory of Euler spaces and show that it is naturally isomorphic to ordinary total homology. From this it follows, for example, that a map from an Euler space is Euler space cobordant to a map from a disjoint union of manifolds.

Stiefel-Whitney homology classes. Recall that if $X$ is a locally compact polyhedron, we can define the link of $x$ in $X, \operatorname{Lk}(x ; X)$ up to p.l. equivalence as the $\operatorname{Lk}(x ; K)$ where $K$ is any triangulation of $X$ having $x$ as a vertex. Recall also that if $K$ is any triangulation of $X$ with $x \in \stackrel{\circ}{ }$ and $A \in K$ with $\operatorname{dim} A=k$ then

$$
\operatorname{Lk}(x ; X) \cong \partial A * \operatorname{Lk}(A ; K) \cong \Sigma^{k} \operatorname{Lk}(A ; K)
$$


where $\cong$ denotes p.l. equivalence, $*$ denotes joining and $\Sigma^{k}$ denotes $k$-fold suspension.

Definition 1. A locally finite simplicial pair $\left(K, K_{0}\right)$ is called an Euler pair if the following equivalent conditions hold:

(a) For $x \in\left|K-K_{0}\right| \chi(\operatorname{Lk}(x ;|K|)) \equiv 0$. For $x \in\left|K_{0}\right|, \chi\left(\operatorname{Lk}\left(x ;\left|K_{0}\right|\right)\right) \equiv$ 0 and $\chi(\operatorname{Lk}(x ;|K|)) \equiv 1$.

(b) For $A \in K-K_{0}, \chi(\operatorname{Lk}(A ; K)) \equiv 0$. For $A \in K_{0}, \chi\left(\operatorname{Lk}\left(A ; K_{0}\right)\right) \equiv 0$ and $\chi(\operatorname{Lk}(A ; K)) \equiv 1$.

Here $\chi$ is the Euler characteristic and $\equiv$ is congruence mod 2 .

A locally compact polyhedral pair is called an Euler pair if some triangulation and hence any triangulation of the pair is Euler. (Note that (a) is p.1. invariant.)

A locally finite simplicial complex $K$ (resp. a locally compact polyhedron $X)$ is called an Euler complex (resp. an Euler space) if the pair $(K, \varnothing)$ (resp. the pair $(X, \varnothing))$ is Euler.

REMARKS. 1. If $X$ is a compact polyhedron then $\chi(\Sigma X) \equiv \chi(X)$. This implies $(\mathrm{a}) \Leftrightarrow(\mathrm{b})$.

2. If $K$ is a finite simplicial complex $\chi(K) \equiv \# K$ (=the number of simplices of $K$ ).

3. If $\left(X, X_{0}\right)$ is Euler then $X-X_{0}$ and $X_{0}$ are Euler. The converse holds if $X_{0} \subset X$ is locally collared. In fact, in this case Remark 1 implies that $X-X_{0}$ Euler is sufficient.

4. If $X$ is a polyhedron, then define

$$
X_{0}=\{x \in X: \chi(\operatorname{Lk}(x ; X)) \equiv 1\} .
$$

$X_{0}$ is a union of open simplices of any triangulation of $X$ and, in fact, is a union of components of open intrinsic skeleta $I^{i}(X)-I^{i-1}(X)$ (cf. [A 1 ; pp. 421, 422]). Clearly, there exists a subpolyhedron $Y$ of $X$ such that $(X, Y)$ is Euler iff $X_{0}$ is closed in $X$, and hence a subpolyhedron iff $\left(X, X_{0}\right)$ is Euler.

All homology is singular homology with $Z_{2}$ coefficients.

Proposition 1. (a) If $K$ is a finite Euler complex and $\eta K$ is a derived subdivision of $K$, then $C_{i}^{\eta}(K)=$ sum of the $i$ dimensional simplices of $\eta K$ is a cycle.

(b) If $\left(K, K_{0}\right)$ is a finite Euler pair, then $C_{i}^{\eta}(K)$ is a relative cycle with boundary $C_{i-1}^{\eta}\left(K_{0}\right)$.

PROoF. If $\hat{\sigma}_{0} \ldots \hat{\sigma}_{i-1}=A$ is an $i-1$ simplex of $\eta K$, where $\sigma_{0}<\cdots<$ $\sigma_{i-1}$ in $K$, then the coefficient of $A$ in $\partial C_{i}^{\eta} \equiv \#$ of $i$-simplices having $A$ as a face, i.e. we must count how many $\sigma$ 's can be inserted into the sequence for $A$ to 
obtain an $i$-simplex. Clearly, this number is equal to

$$
\# \partial \sigma_{0}+\sum_{j=1}^{i-1} \# \operatorname{Lk}\left(\sigma_{j-1}, \partial \sigma_{j}\right)+\# \operatorname{Lk}\left(\sigma_{i-1}, K\right) .
$$

Since the Euler number of a sphere is 0 mod 2, this coefficient is $\equiv \chi\left(\operatorname{Lk}\left(\sigma_{i-1} K\right)\right)$.

So if $\left(K, K_{0}\right)$ is Euler this coefficient is 0 if $\sigma_{i-1} \in K-K_{0}$, i.e. if $A \in$ $\eta K-\eta K_{0}$ and is 1 if $\sigma_{i-1} \in K_{0}$, i.e. if $A \in \eta K_{0}$.

REMARK. If $\eta K$ and $\bar{\eta} K$ are two different derived subdivisions of $K$ obtained by starring the simplices of $K$ at different internal points, then $\hat{\sigma} \rightarrow \bar{\sigma}$, the obvious simplicial isomorphism carries $C_{i}^{\eta}\left(K, K_{0}\right)$ to $C_{i}^{\bar{\eta}}\left(K, K_{0}\right)$. The map is isotopic to the identity so the chains represent the same homology class. So we will drop the superscript $\eta$.

Definition 2. If $\left(K, K_{0}\right)$ is a finite Euler pair, then $w_{i}\left(K, K_{0}\right)$ is the homology class of $C_{i}\left(K, K_{0}\right)$.

PROPOSITION 2. The $w_{i}$ 's are p.l. invariants, i.e. if $\left(K, K_{0}\right)$ and $\left(L, L_{0}\right)$ are two triangulations of the polyhedral Euler pair $\left(X, X_{0}\right)$ then $w_{i}\left(K, K_{0}\right)=$ $w_{i}\left(L, L_{0}\right)$ in $H_{i}\left(X, X_{0}\right)$ and so we can write $w_{i}\left(X, X_{0}\right)$ for these classes.

Proof. If we subdivide $K$ by a stellar subdivision at $\tau \in K$, we obtain $K^{*}$ which is the same complex as $K$ on $\left|K-\mathrm{St}^{0}(\tau ; K)\right|$. Choose derived $\eta K$ and $\eta K^{*}$ which agree there. We note that as singular chains the difference $C_{i}{ }^{\eta}(K)-$ $C_{i}^{\eta}\left(K^{*}\right)$ is carried by $|\mathrm{St}(\tau, K)|$.

If $\tau \in K-K_{0}$ then $C_{i-1}^{\eta}\left(K_{0}\right)=C_{i-1}^{\eta}\left(K_{0}^{*}\right)$ and so $C_{i}^{\eta}(K)-C_{i}^{\eta}\left(K^{*}\right)$ is a cycle on $K$ carried by the contractible space $|\operatorname{St}(\tau ; K)|$.

If $\tau \in K_{0}$ then $C_{i}^{\eta}(K)-C_{i}^{\eta}\left(K^{*}\right)$ is a relative cycle carried by the contractible pair $\left(\operatorname{St}(\tau ; K), \operatorname{St}\left(\tau ; K_{0}\right)\right)$.

Now if $K$ and $L$ have a common subdivision it is not known whether they have a common stellar subdivision, but it is known e.g. [G, p. 41, Theorem II-17] that one can get from one to the other by a finite sequence of stellar subdivisions or inverse stellar subdivisions i.e. there exists $K=K_{0}, K_{1}, \ldots, K_{n}=$ $L$ such that for $i=0, \ldots, n-1$ either $K_{i}$ is a stellar subdivision of $K_{i+1}$ of $K_{i+1}$ is a stellar subdivision of $K_{i}$. Note that any triangulation of $X$ is a triangulation of the pair $\left(X, X_{0}\right)$ because $\chi(\operatorname{Lk}(x ; X)) \equiv 0$ for $x \notin X_{0}$ and $\equiv 1$ for $x \in X_{0}$.

Remark. If $\partial: H_{i}\left(X, X_{0}\right) \rightarrow H_{i-1}\left(X_{0}\right)$, Propositions 1 and 2 imply that $\partial w_{i}\left(X, X_{0}\right)=w_{i-1}\left(X_{0}\right)$.

Proposition 3 (Excision). Let $X_{0}, X_{1}, X_{2}, X_{3}$ be closed subpolyhedra of a compact polyhedron $X$ such that $X=X_{1} \cup X_{2}, X_{0} \subset X_{3} \cup X_{2}$ and $\left(X, X_{3}\right)$ 
and $\left(X_{1}, X_{0}\right)$ are Euler pairs; then in the diagram:

$$
\begin{gathered}
H_{i}\left(X_{1}, X_{0}\right) \stackrel{j_{1} *}{\longrightarrow} H_{i}\left(X, X_{3} \cup X_{2}\right) \stackrel{j *}{\stackrel{j}{L}} H_{i}\left(X, X_{3}\right), \\
j_{1} *\left(w_{i}\left(X_{1}, X_{0}\right)\right)=j *\left(w_{i}\left(X, X_{3}\right)\right) .
\end{gathered}
$$

Proof. Triangulate to obtain $K$ a complex on $X$ and $K_{i}$ with $\left|K_{i}\right|=X_{i}$, $i=0,1,2,3 . C_{i}^{\eta}(K)-C_{i}^{\eta}\left(K_{1}\right)$ is carried by $X_{2}$, i.e. if $\partial_{0} \ldots \partial_{i} \in \eta K-\eta K_{1}$ then $\sigma_{i} \in K_{2}$ and $\partial_{0} \ldots \partial_{i}$ is in $X_{2}$.

Letting $X_{3}=\varnothing$ we obtain:

COROLlaRY. Let $X_{0}, X_{1}, X_{2}$ be closed subpolyhedra of a compact Euler space $X$ such that $X=X_{1} \cup X_{2}, X_{0} \subset X_{2}$ and $\left(X_{1}, X_{0}\right)$ is an Euler pair, then in the diagram

$$
\begin{gathered}
H_{i}\left(X_{1}, X_{0}\right) \stackrel{j_{1} *}{\longrightarrow} H_{i}\left(X, X_{2}\right) \stackrel{j *}{\longleftarrow} H_{i}(X), \\
j_{1} * w_{i}\left(X_{1}, X_{0}\right)=j * w_{i}(X) .
\end{gathered}
$$

Proposition 4 (MAyer-Vietoris). Let $X_{0}, X_{1}, X_{2}, X_{3}$ be closed subpolyhedra of a compact polyhedron $X$ such that $X_{0}=X_{1} \cap X_{2}, X_{1} \cup X_{2}=X$ and $\left(X_{1}, X_{0} \cup\left(X_{3} \cap X_{1}\right)\right),\left(X_{2}, X_{0} \cup\left(X_{3} \cap X_{2}\right)\right)$ and $\left(X_{0}, X_{0} \cap X_{3}\right)$ are Euler pairs.

Then $\left(X_{3} \cap X_{2}, X_{3} \cap X_{0}\right),\left(X_{3} \cap X_{1}, X_{3} \cap X_{0}\right)$ and $\left(X, X_{3}\right)$ are Euler pairs, and the Mayer-Vietoris boundary $\Delta: H_{i}\left(X, X_{3}\right) \rightarrow H_{i-1}\left(X_{0}, X_{0} \cap X_{3}\right)$ takes $w_{i}\left(X, X_{3}\right)$ to $w_{i-1}\left(X_{0}, X_{0} \cap X_{3}\right)$.

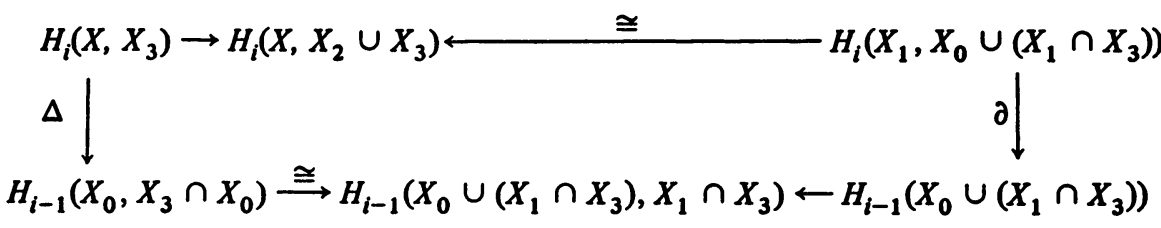

Proof. To see that $\left(X_{3} \cap X_{i}, X_{3} \cap X_{0}\right), i=1,2$, and $\left(X, X_{3}\right)$ are Euler pairs we look first at points $x \in X_{0} \cap X_{3} . \operatorname{Lk}\left(x ;\left(X_{3} \cap X_{i}\right) \cup X_{0}\right)=$ $\operatorname{Lk}\left(x ; X_{3} \cap X_{i}\right) \cup \operatorname{Lk}\left(x ; X_{0}\right)$ with intersection $\operatorname{Lk}\left(x ; X_{0} \cap X_{3}\right)$. So $\chi\left(\operatorname{Lk}\left(x ; X_{3} \cap X_{i}\right)\right) \equiv 1 . \operatorname{Lk}(x ; X)=\mathrm{Lk}\left(x ; X_{1}\right) \cup \mathrm{Lk}\left(x ; X_{2}\right)$ with intersection $\mathrm{Lk}\left(x ; X_{0}\right)$ so $\chi(\mathrm{Lk}(x ; X)) \equiv 1$. For $x \in X_{0}-X_{3}, \mathrm{Lk}(x ; X)=\mathrm{Lk}\left(x ; X_{1}\right) \cup$ $\mathrm{Lk}\left(x ; X_{2}\right)$ with intersection $\mathrm{Lk}\left(x ; X_{0}\right)$ implies that $\chi(\mathrm{Lk}(x ; X)) \equiv 0$.

That $\Delta w_{i}\left(X, X_{3}\right)=w_{i-1}\left(X_{0}, X_{3} \cap X_{0}\right)$ follows from Proposition 3, the remark after Proposition 2, and the Corollary to Proposition 3, via the commutative diagram above. 
Letting $X_{\mathbf{3}}=\varnothing$ we obtain:

Corollary 1. Let $X_{0}, X_{1}, X_{2}$ be closed subpolyhedra of a compact polyhedron $X$ such that $X_{0}=X_{1} \cap X_{2}, X=X_{1} \cup X_{2}$ and $\left(X_{i}, X_{0}\right)(i=1,2)$ are Euler pairs.

Then $X$ is an Euler space and $\Delta w_{i}(X)=w_{i-1}\left(X_{0}\right)$ where $\Delta$ is the MayerVietoris boundary:

$$
H_{i}(X) \rightarrow H_{i}\left(X, X_{2}\right) \stackrel{\cong}{\cong} H_{i}\left(X_{1}, X_{0}\right) \stackrel{\partial}{\longrightarrow} H_{i-1}\left(X_{0}\right) .
$$

Corollary 2. Let $\left(X, X_{0}\right)$ be a compact Euler pair and $(I, \dot{I})=$ $([0,1],\{0,1\})$, then $\left(X \times I, X \times I \cup X_{0} \times I\right)$ is an Euler pair and

where $\bar{\Delta}$ is given by:

$$
\bar{\Delta} w_{i}\left(X \times I, X \times I \cup X_{0} \times I\right)=w_{i-1}\left(X, X_{0}\right)
$$

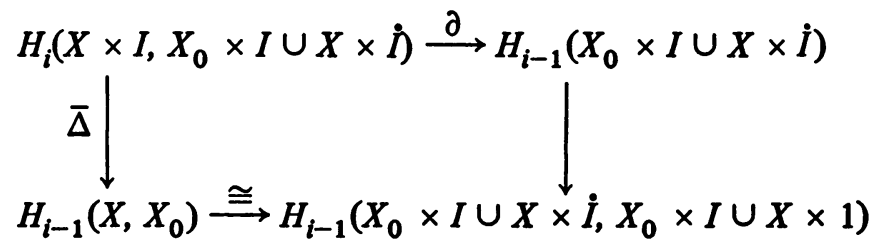

Proof. Let $\bar{X}_{1}=X \times[0,1 / 2], \bar{X}_{2}=X \times[1 / 2,1], \bar{X}_{3}=X_{0} \times I \cup X \times \dot{I}$, $\bar{X}_{0}=X \times 1 / 2$ and $\bar{X}=X \times I$. Using the addition formula for Euler characteristics again we see that Proposition 4 applies to this situation. It remains to identify $\Delta$ with $\bar{\Delta}$, which is easy using a map $r: I \rightarrow I$ with $r[0,1 / 2]=0$ and $r(1)=1$.

Remark. Since $(X \times 0, X \times 0) \subset\left(\bar{X}_{1}, \bar{X}_{3} \cap \bar{X}_{1}\right)$ and $(X \times 1, X \times 1) \subset$ $\left(\bar{X}_{2}, \bar{X}_{2} \cap \bar{X}_{3}\right)$ are homotopy equivalences, exactness of the Mayer-Vietoris sequence implies that $\bar{\Delta}$ is an isomorphism.

Corollary 3. Let $X$ be a compact Euler space with $\chi(X) \equiv 0$; then $\Sigma X$ is an Euler space and $(c X, X)$ is an Euler pair. The suspension isomorphism, $\sigma: H_{i}(X) \rightarrow H_{i-1}(\Sigma X)$ takes $w_{i}(X)$ to $w_{i+1}(\Sigma X)$, where $\sigma$ is the inverse to the Mayer-Vietoris boundary for $\left(\Sigma X ; c_{1} X, c_{2} X\right)$ (recall that the suspension is the union of two cones on $X$ intersecting in their common base).

Now we turn to the relation between these homology classes and mappings. Another way of stating Proposition 2 is that if $f:\left(X, X_{0}\right) \rightarrow\left(Y, Y_{0}\right)$ is a pl. homeomorphism with $\left(X, X_{0}\right)$ (and hence $\left(Y, Y_{0}\right)$ also) an Euler pair, then $f_{*}\left(w_{i}\left(X, X_{0}\right)\right)=w_{i}\left(Y, Y_{0}\right)$, where $f_{*}$ is the induced map on $Z_{2}$ homology.

This is pretty weak invariance. It is reasonable to suppose that the $w_{i}$ 's are homotopy invariants. This is true, after all, of the Stiefel-Whitney classes on manifolds.

However, it is not true for Euler spaces. In fact let $X=S^{2} \vee S^{2}$, i.e. the 
union of two spheres along a point and let $f: X \rightarrow X$ by the union of $s: S^{2}$ $\rightarrow S^{2} \vee S^{2}$ and $i_{2}: S^{2} \rightarrow S^{2} \vee S^{2}$ on the two pieces of the domain, $s$ is the coproduct obtained by pinching the equator to a point and $i_{2}$ is the inclusion on the second term. $H_{2}(X)=Z_{2} \oplus Z_{2}$ generated by $z_{1}$ and $z_{2}$, the fundamental classes on the two spheres. $w_{2}(X)=z_{1}+z_{2}$ and so $f_{*} w_{2}(X)=s_{*}\left(z_{1}\right)+i_{2} *\left(z_{2}\right)$ $=z_{1}+z_{2}+z_{2}=z_{1}$. Thus $f_{*} w_{2}(X) \neq w_{2}(X)$. Note that $X$ is an Euler space as the link of the bad point is the disjoint union of two circles.

In the positive direction we have invariance under the appropriate notion of cobordism of maps.

DEFinition 3. Let $\left(X, X_{0}\right),\left(Y, Y_{0}\right)$ be compact Euler pairs and $\left(T, T_{0}\right)$ any topological pair, and let $f:\left(X, X_{0}\right) \rightarrow\left(T, T_{0}\right)$ and $g:\left(Y, Y_{0}\right) \rightarrow\left(T, T_{0}\right)$ be continuous.

A cobordism between $f$ and $g$ is a quintuple $\left(W, W_{0}, W_{1}, i_{X}, i_{Y}\right)$ with $W \supset$ $W_{0} \supset W_{1}$ compact polyhedra, $i_{X}: X \rightarrow W_{0}, i_{Y}: Y \rightarrow W_{0}$ p.l. embeddings and a map $F:\left(W, W_{1}\right) \rightarrow\left(T, T_{0}\right)$ such that

(1) $i_{X}(X) \cap w_{1}=i_{X}\left(X_{0}\right), i_{Y}(Y) \cap w_{1}=i_{Y}\left(Y_{0}\right), i_{X}(X) \cap i_{Y}(Y)=\varnothing$ and $W_{0}=i_{X}(X) \cup W_{1} \cup i_{Y}(Y)$.

(2) $\left(W, W_{0}\right)$ and $\left(W_{1}, i_{X}\left(X_{0}\right) \cup i_{Y}\left(Y_{0}\right)\right)$ are Euler pairs.

(3) $F \cdot i_{X}=f$ and $F \cdot i_{Y}=g$.

REMARK. Clearly, if $f$ and $g$ are homotopic then they are cobordant, see Corollary 2 of Proposition 4.

Proposition 5. If $f:\left(X, X_{0}\right) \rightarrow\left(T, T_{0}\right)$ and $g:\left(Y, Y_{0}\right) \rightarrow\left(T, T_{0}\right)$ be cobordant. Then

$$
f_{*} w_{i}\left(X, X_{0}\right)=g_{*} w_{i}\left(Y, Y_{0}\right) \text { for all } i
$$

Proof. Using p.l. invariance of the $w_{i}$ 's we shall regard $i_{X}$ and $i_{Y}$ as inclusions. We consider the map of the triples induced by $F:\left(W, W_{0}, W_{1}\right) \rightarrow$ $\left(T, T, T_{0}\right)$.

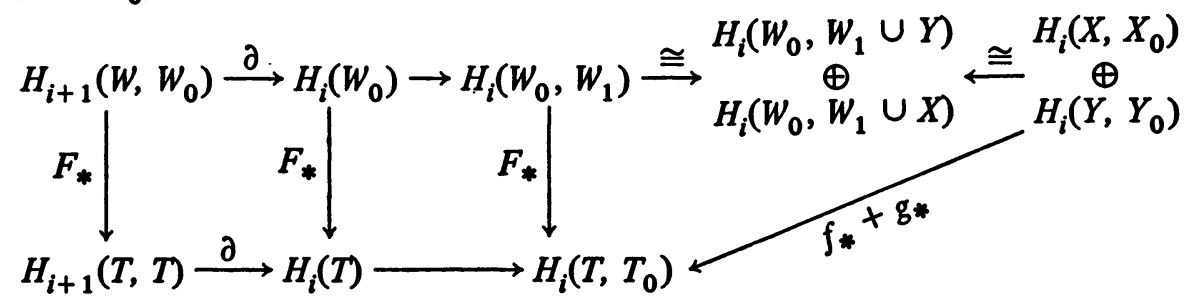

Note that $\left(X \cup Y, X_{0} \cup Y_{0}\right) \subset\left(W_{0}, W_{1}\right),\left(X, X_{0}\right) \subset\left(W_{0}, \dot{W}_{1} \cup Y\right)$ and $\left(Y, Y_{0}\right) \subset\left(W_{0}, W_{1} \cup X\right)$ are excisions. Now by the Corollary to Proposition 3 applied twice, $w_{i}\left(W_{0}\right)$ on the one hand and $w_{i}\left(X, X_{0}\right)+w_{i}\left(Y, Y_{0}\right)$ on the other, 
map to the same thing in $H_{i}\left(W_{0}, W_{1} \cup Y\right) \oplus H_{i}\left(W_{0}, W_{1} \cup X\right)$. Since $\partial w_{i+1}\left(W, W_{0}\right)$ $=w_{i}\left(W_{0}\right)$ and since $H_{*}(T, T)=0$, it follows that $f_{*} w_{i}\left(X, X_{0}\right)+g_{*} w_{i}\left(Y, Y_{0}\right)=$ 0. So $f_{*} w_{i}\left(X, X_{0}\right)=g_{*} w_{i}\left(Y, Y_{0}\right)$.

As an illustration we will look at an example.

Definition 4. Let $f: X_{1} \rightarrow X_{2}$ be a proper p.l. map, of Euler spaces, with $f\left(X_{1}\right)=X_{2}$.

(a) Call $f$ even if, for all $x \in X_{2}, \chi\left(f^{-1}(x)\right) \equiv 0$.

(b) Call $f$ odd if, for all $x \in X_{2}, \chi\left(f^{-1}(x)\right) \equiv 1$.

We will show that (a) if $f: X_{1} \rightarrow X_{2}$ is odd then $f$ is cobordant to $1_{X_{2}}$, and (b) if $f$ is even then $f$ is cobordant to $\varnothing: \varnothing \rightarrow X_{2}$, the empty map. It follows that odd maps preserve the Stiefel-Whitney homology classes and even maps kill them.

We will use material on general complexes and mapping cylinders as discussed in $\left[\mathbf{A}_{2}\right]$.

LemmA. Let $\mathfrak{D}_{i}$ be finite general complexes on compact polyhedra $X_{i}, i=$ 1,2 , and $\gamma: D_{1} \rightarrow \mathfrak{D}_{2}$ an isomorphism of general complexes. Assume that $K$ is a constant such that $\chi(\gamma(\sigma))=K \chi(\sigma)$ for all $\sigma \in \mathfrak{D}_{1}$. Then $\chi\left(X_{2}\right)=K \chi\left(X_{1}\right)$.

Proof. By induction on the number of elements in the complexes. Let $\sigma$ be a free element of $\mathfrak{D}_{1}$, i.e. $\sigma^{\prime} \in \mathfrak{D}_{1}$ implies $\sigma^{\prime} \cap \sigma \subset \partial \sigma$ or $\sigma^{\prime}=\sigma$, then $\gamma \sigma$ is free in $\mathfrak{D}_{2}$. Let $\mathfrak{D}_{1}^{\prime}=\mathfrak{D}_{1}-\sigma, \mathfrak{D}_{2}^{\prime}=\mathfrak{D}_{2}-\gamma \sigma, \mathfrak{B}_{1}=$ all faces of $\left.\sigma\right\} \cup\{\sigma\}$, $\mathfrak{B}_{2}=\{$ all faces of $\gamma \sigma\} \cup\{\gamma \sigma\}$.

If $\mathscr{B}_{i}=\mathfrak{D}_{i}$ then $\chi\left(X_{2}\right)=\chi(\gamma \sigma)=K \chi(\sigma)=K \chi\left(X_{1}\right)$.

If $\mathfrak{B}_{i}$ and $\mathfrak{D}_{i}^{\prime}$ are proper subsets of $\mathfrak{D}_{i}, i=1,2$, then by induction hypothesis $\chi\left(\left|\mathscr{B}_{2}\right|\right)=K \chi\left(\left|\mathfrak{B}_{1}\right|\right), \quad \chi\left(\left|\mathfrak{D}_{2}^{\prime}\right|\right)=K \chi\left(\left|\mathfrak{D}_{1}^{\prime}\right|\right)$ and $\chi\left(\mathfrak{B}_{2}|\cap| \mathfrak{D}_{2}^{\prime} \mid\right)=\chi\left(\mathfrak{B}_{2} \cap \mathfrak{D}_{2}^{\prime} \mid\right)$ $=K \chi\left(\mathscr{B}_{1} \cap \mathfrak{D}_{1}^{\prime} \mid\right)=K \chi\left(\left|\mathbb{B}_{1}\right| \cap\left|\mathfrak{D}_{1}^{\prime}\right|\right)$; and so the result follows from the addition formula for Euler characteristic.

REMARK. In the above proof and hence in the above theorem "=" can be replaced by “@mod $n$ " for any $n$.

This lemma is motivated by and can be used to prove the well-known fact that if $\pi: E \rightarrow B$ is a p.l. fibration with $E$ compact and fibre $F$, then $\chi(E)=$ $\chi(F) \chi(B)$. In particular if $E$ admits a $Z_{2}$ symmetry, then $\chi(E) \equiv 0$. Thus, for a polyhedron to be Euler it is sufficient that the links of points admit p.1. $Z_{2}$ symmetries.

Given $f: K_{1} \rightarrow K_{2}$ simplicial map either even or odd, of locally finite Euler complexes, we examine the simplicial mapping cylinder $C_{f}$. We utilize the general complexes on $K_{1}, K_{2}$ and $C_{f}$ given by the map developed by Cohen and discussed in $\left[\mathrm{A}_{2}, \mathrm{pp} .404,407-408,410\right]$. For $\sigma \in K_{2}, \mathrm{Lk}\left(\sigma ; C_{f}\right) \cong \dot{Q}(\sigma ; f) \cup D(\sigma ; f)$ (intersection $\dot{D}(\sigma, f)$ ). The map $Q\left(\sigma^{\prime} ; f\right) \rightarrow D\left(\sigma^{\prime} ; K_{2}\right)$ is an isomorphism of 
general complexes on $\dot{Q}(\sigma ; f)$ and $\dot{D}\left(\sigma ; K_{2}\right)$ as $\sigma^{\prime}$ varies with $\sigma<\sigma^{\prime}$. Thus, by the above lemma $\chi(\dot{Q}(\sigma ; f))=\chi\left(\dot{D}\left(\sigma ; K_{2}\right)\right) \equiv 0$.

Now $D(\sigma ; f)$ is a regular neighborhood of $f^{-1}(\hat{\sigma})$ and so $\chi(D(\sigma ; f)) \equiv \epsilon$ where $\epsilon=0$ or 1 according as $f$ is even or odd. The map $D\left(\sigma^{\prime} ; f\right) \rightarrow D\left(\sigma^{\prime} ; K_{2}\right)$ with $\sigma^{\prime}>\sigma$ is an isomorphism of general complexes on $\dot{D}(\sigma ; f)$ and $\dot{D}\left(\sigma ; K_{2}\right)$ and hence by the lemma $\chi(\dot{D}(\sigma ; f)) \equiv \epsilon \chi\left(\dot{D}\left(\sigma, K_{2}\right)\right) \equiv 0$.

Thus by the addition formula for Euler characteristics we have $\chi\left(\operatorname{Lk}\left(\sigma ; C_{f}\right)\right)$ $\equiv \chi(D(\sigma ; f)) \equiv \epsilon$.

Thus, for points $x \in\left|K_{2}\right|$,

$$
\chi\left(\operatorname{Lk}\left(x ; C_{f}\right)\right) \equiv \epsilon \quad \text { and } \quad \chi\left(\operatorname{Lk}\left(x ; K_{2}\right)\right) \equiv 0
$$

For $x \in\left|K_{1}\right|$,

$$
\chi\left(\operatorname{Lk}\left(x ; C_{f}\right)\right) \equiv 1 \text { and } \chi\left(\operatorname{Lk}\left(x ; K_{1}\right)\right) \equiv 0
$$

as $K_{1}$ is collared in $C_{f}$. For $x \in C_{f}-\left|K_{1}\right| \cup\left|K_{2}\right|, \chi\left(\operatorname{Lk}\left(x ; C_{f}\right)\right) \equiv 0$ because the collar can be extended to include $x$ so that such a point, looking locally, thinks it is inside $K_{1} \times I-K_{1} \times I$ which is Euler.

In short, if $f$ is even $\left(C_{f},\left|K_{1}\right|\right)$ is an Euler pair and if $f$ is odd $\left(C_{f},\left|K_{1}\right| \cup\right.$ $\left.\left|K_{2}\right|\right)$ is an Euler pair.

We can also show the converse, more precisely we show that if $\chi\left(\operatorname{Lk}\left(\sigma, C_{f}\right)\right)$ is the same $(\bmod 2)$ for. all $\sigma \in K_{2}$ then $\chi\left(f^{-1}(\eta \sigma)\right)$ is the same for all $\sigma \in K_{2}$.

Note that the above proof shows that if $\chi\left(f^{-1} \eta\left(\sigma^{\prime}\right)\right)$ is constant mod 2 for all $\sigma^{\prime}>\sigma$, then $\chi\left(\operatorname{Lk}\left(\sigma ; C_{f}\right)\right) \equiv \chi\left(f^{-1}(\eta \sigma)\right)$.

Now, assume $K_{2}$ is finite dimensional and let $n$ be the maximum codimension such that $\chi\left(f^{-1}(\eta \sigma)\right)$ is constant $\bmod 2$ for $\sigma$ of codimension $<n$. So if $\chi\left(f^{-1}(\eta \sigma)\right)$ is not constant for all $\sigma$, then there exist $\sigma_{1}$ and $\sigma_{2}$ of codimension $\leqslant n$ such that $\chi\left(f^{-1}\left(\eta \sigma_{1}\right)\right) \neq \equiv\left(f^{-1}\left(\eta \sigma_{2}\right)\right)$. Since $\sigma^{\prime}>\sigma_{i}$ for $i=1$ or 2 implies codimension $\sigma^{\prime}<n$ it follows that

$$
\chi\left(\operatorname{Lk}\left(\sigma_{1} ; C_{f}\right)\right) \neq \chi\left(\operatorname{Lk}\left(\sigma_{2} ; C_{f}\right)\right)
$$

If $K_{2}$ is not finite dimensional then choose $\sigma_{1}$ and $\sigma_{2}$ with $\chi\left(f^{-1}\left(\eta \sigma_{1}\right)\right) \neq$ $\chi\left(f^{-1}\left(\eta \sigma_{2}\right)\right)$ and apply the above inductive argument on codimension to the simplices interior to $\operatorname{St}\left(\sigma_{1} ; K_{2}\right) \cup \operatorname{St}\left(\sigma_{2} ; K_{2}\right)$, noting that, since $K_{2}$ is locally finite, this is a finite complex.

In sum we have proved:

Proposition 6. Let $f: X_{1} \rightarrow X_{2}$ be an onto, proper p.l. map of Euler spaces and let $C_{f}$ be a mapping cylinder obtained by triangulating $f$.

(a) $\left(C_{f}, X_{1}\right)$ is an Euler pair iff $f$ is an even map.

(b) $\left(C_{f}, X_{1} \cup X_{2}\right)$ is an Euler pair iff $f$ is an odd map. 
Proof. If either $\left(C_{f}, X_{1}\right)$ or $\left(C_{f}, X_{1} \cup X_{2}\right)$ is an Euler pair then $\chi\left(\operatorname{Lk}\left(x ; C_{f}\right)\right)$ is constant for $x$ in $X_{2}$ and so $\chi\left(f^{-1}(x)\right)$ is constant for $x \in X_{2}$ so $f$ is either even or odd. Since $\chi\left(\operatorname{Lk}\left(\sigma ; C_{f}\right)\right) \equiv \chi\left(f^{-1}(\eta \sigma)\right)$ in these cases, the possibilities pair up properly.

In private correspondence, McCrory has shown me a proof of this result which requires only that the mapping cylinder of $f$ be triangulable with pointinverses as subcomplexes, instead of requiring that $f$ itself be a p.l. map.

Let $p_{f}: C_{f} \rightarrow X_{2}$ be any retraction extending $f$ on $X_{1}$.

Corollary 1. (a) If $f: X_{1} \rightarrow X_{2}$ is an even map then $\left(C_{f}, X_{1}, \varnothing\right.$; inclusion $\left._{X_{1}}, \varnothing\right)$ and the map $p_{f}$ is a cobordism of $f$ and the empty map.

(b) If $f: X_{1} \rightarrow X_{2}$ is an odd map then $\left(C_{f}, X_{1} \cup X_{2}, \varnothing\right.$; inclusion $X_{1}$, inclusion $X_{X_{2}}$ ) and the map $p_{f}$ is a cobordism of $f$ and $1_{X_{2}}$.

Corollary 2. (a) If $f: X_{1} \rightarrow X_{2}$ is an even map then $f_{*} w_{i}\left(X_{1}\right)=0$ for all $i$.

(b) If $f: X_{1} \rightarrow X_{2}$ is an odd map then $f_{*} w_{i}\left(X_{1}\right)=w_{i}\left(X_{2}\right)$ for all $i$.

$Z_{2}$-bordism theories. Let $\mathcal{F}^{n}, n \in Z$, be a sequence of classes of compact pl. pairs satisfying the following four axioms (write $X \in \mathfrak{F}^{n}$ for $(X, \varnothing) \in \mathfrak{F}^{n}$ ):

Axiom 1. If $\left(X, X_{0}\right) \cong\left(Y, Y_{0}\right)$ and $\left(X, X_{0}\right) \in \mathcal{F}^{n}$ then $\left(Y, Y_{0}\right) \in \mathcal{F}^{n}$.

Axiom 2. $\left(X, X_{0}\right) \in \mathfrak{F}^{n}$ implies $X_{0} \in \mathcal{F}^{n-1}$ and $\left(X \times I, X \times I \cup X_{0} \times I\right)$ $\in \mathfrak{F}^{n+1}$.

Axiom 3 (Cutting). Let $\left(X, X_{0}\right) \in \mathcal{F}^{n}, A$ a closed subpolyhedron of $X$, $\left(V, V_{0}\right)$ a regular neighborhood pair for $A$ in $\left(X, X_{0}\right)$. If $Y=\overline{X-V}$ and $Y_{0}=$ $\overline{X_{0}-V_{0}}$, then $\left(Y, \dot{V} \cup Y_{0}\right) \in \mathfrak{F}^{n},\left(V, \dot{V} \cup V_{0}\right) \in \mathfrak{F}^{n}$ and $\left(\dot{V}, \dot{V} \cap Y_{0}\right) \in \mathfrak{F}^{n-1}$

Axiom 4 (PAsting). Let $X \supset X_{0} \supset X_{1}, Y \supset Y_{0} \supset Y_{1}$ and $Z \supset Z_{0} \supset \varnothing$ be pl. triples with $X$ and $Y$ disjoint and $i_{X}: Z \rightarrow X_{0}, i_{Y}: Z \rightarrow Y_{0}$ p.l. embeddings such that

(a) $X_{0}=i_{X}(Z) \cup X_{1}, \quad Y_{0}=i_{Y}(Z) \cup X_{1}$ and $i_{X}^{-1}\left(X_{1}\right)=i_{Y}^{-1}\left(Y_{1}\right)=Z_{0}$.

(b) If $\left(X, X_{0}\right),\left(Y, Y_{0}\right) \in \mathcal{F}^{n}$ and $\left(X_{1}, i_{X}\left(Z_{0}\right)\right),\left(Y_{1}, i_{Y}\left(Z_{0}\right)\right),\left(Z, Z_{0}\right) \in$ $\mathfrak{F}^{n-1}$, then $\left(X \cup \cup_{Z} Y, X_{1} \cup \cup_{0} Y_{1}\right) \in \mathfrak{F}^{n}$.

Remarks. 1. Letting $A=X$ in 3 implies $\varnothing \in \mathcal{F}^{n}$ provided $\mathcal{F}^{n}$ is not empty. For convenience we will assume in addition $\varnothing \in \mathcal{F}^{n}$ for all $n$.

2. For the definition and theory of regular neighborhood pairs we refer the reader to Cohen's paper [C]. In particular, if $\left(V, V_{0}\right)$ is a regular neighborhood pair of $A$ in $\left(X, X_{0}\right)$, then $V_{0}$ is a regular neighborhood of $A \cap X_{0}$ in $X_{0}$. Hence, in Axiom 3, it follows that $\left(Y_{0}, Y_{0} \cap \dot{V}\right),\left(V_{0}, V_{0} \cap \dot{V}\right) \in \mathcal{F}^{n-1}$ by Axiom 3 applied to $A \cap X_{0}$ in $\left(X_{0}, \varnothing\right)$. We recall that $\dot{V}_{0}=\dot{V} \cap X_{0}=V_{0} \cap Y_{0}=V_{0} \cap$ $\dot{V}=\dot{V} \cap Y_{0}$ from the simplicial definitions [C, pp. 192-194]. Also, $\left(\dot{V}, \dot{V}_{0}\right)$ is 
collared in $\left(V, V_{0}\right)$ and $\left(Y, Y_{0}\right)$, and bicollared in $\left(X, X_{0}\right)$. This relative version of [C, p. 203, Theorem 5.3] does not seem to appear anywhere. The proof is the same as Cohen's absolute theorem, except that the equation on p. 203 of [C] where a common factor $S$ is pulled off of various links becomes a pair $\left(S, S_{0}\right)$ pulled from link pairs. Then one applies the relative theorem $\left[\mathrm{A}_{1}\right.$, p. 455 , Theorem 2], in place of Cohen's theorem [C, p. 199, Proposition 4.2].

Definition 5. (a) Let $\left(X, X_{0}\right)$ and $\left(Y, Y_{0}\right) \in \mathfrak{F}^{n}$; then a cobordism $\mathcal{A}$ between $\left(X, X_{0}\right)$ and $\left(Y, Y_{0}\right)$ is a quintuple $\left(W, W_{0}, W_{1} ; i_{X}, i_{Y}\right)$ where $W \supset W_{0}$ $\supset W_{1}$ and $i_{X}: X \rightarrow W_{0}$ and $i_{Y}: Y \rightarrow W_{0}$ are p.l. embeddings, satisfying:

(1) $i_{X}(X) \cap w_{1}=i_{X}\left(X_{0}\right), i_{Y}(Y) \cap w_{1}=i_{Y}\left(Y_{0}\right), i_{X}(X) \cap i_{Y}(Y)=\varnothing$ and $w_{0}=i_{X}(X) \cup w_{1} \cup i_{Y}(Y)$.

(2) $\left(W, W_{0}\right) \in \mathfrak{F}^{n+1}$ and $\left(W_{1}, i_{X}\left(X_{0}\right) \cup i_{Y}\left(Y_{0}\right)\right) \in \mathfrak{F}^{n}$.

(b) Let $\left(X, X_{0}\right)$ and $\left(Y, Y_{0}\right) \in \mathcal{F}^{n}$. Let $\left(X, X_{0}\right)$ and $\left(Y, Y_{0}\right) \in \mathcal{F}^{n}$. Let $f:\left(X, X_{0}\right) \rightarrow\left(T, T_{0}\right)$ and $g:\left(Y, Y_{0}\right) \rightarrow\left(T, T_{0}\right)$ be continuous maps to a topological pair $\left(T, T_{0}\right)\left(T_{0}\right.$ is closed in $\left.T\right)$, then a cobordism $V$ between $f$ and $g$ is a pair $(\mathcal{A}, F)$ where $\mathfrak{A}=\left(W, W_{0}, W_{1} ; i_{X}, i_{Y}\right)$ is a cobordism between $\left(X, X_{0}\right)$ and $\left(Y, Y_{0}\right)$ and $F:\left(W, W_{1}\right) \rightarrow\left(T, T_{0}\right)$ is a continuous map such that $F \cdot i_{X}=$ $f$ and $F \cdot i_{Y}=g$.

With slight abuse of notation we shall usually regard $i_{X}$ and $i_{Y}$ as inclusions and write $\mathcal{A}=\left(W, W_{0}, W_{1}\right)$ when $i_{X}$ and $i_{Y}$ are clear.

By Axiom 2, the cobordism relations are reflexive and by the pasting axiom they are transitive. They are clearly symmetric and hence are equivalence relations. We denote by $\Omega_{n}\left(T, T_{0}\right)$ the set of equivalence classes of maps $f:\left(X, X_{0}\right) \rightarrow\left(T, T_{0}\right)$ $\left(X, X_{0}\right) \in \mathcal{F}^{n}$ (equivalence is cobordism of maps). Under disjoint union (possible by pasting with $Z=\varnothing)$ this is a group with zero element $\varnothing:(\varnothing, \varnothing) \rightarrow\left(T, T_{0}\right)$ and satisfying $f+f=0$ by the cobordism $\left(\left(X \times I, X \times I \cup X_{0} \times I, X_{0} \times I\right)\right.$, $\left.f \cdot \pi_{X}\right)$.

The boundary $\partial: \Omega_{n}\left(T, T_{0}\right) \rightarrow \Omega_{n-1}\left(T_{0}\right)$ is defined by $\partial[f]=\left[f \mid X_{0}\right]$ where $f:\left(X, X_{0}\right) \rightarrow\left(T, T_{0}\right)$.

If $F:\left(T, T_{0}\right) \rightarrow\left(R, R_{0}\right)$ is continuous then $F_{*}[f]=[F \cdot f]$ defines $F_{*}: \Omega_{n}\left(T, T_{0}\right) \rightarrow \Omega_{n}\left(R, R_{0}\right)$.

PROPOSITION 7. $\Omega_{*}$ is a homology theory.

Proof. The homotopy axiom is clear using the usual product cobordism.

Excision. Let $U$ be an open set of $T$ with $\bar{U} \subset$ interior $T_{0}$. Then we show that $\Omega_{n}\left(T-U, T_{0}-U\right) \stackrel{i_{*}}{\longrightarrow} \Omega_{n}\left(T, T_{0}\right)$ is an isomorphism.

Let $f:\left(X, X_{0}\right) \rightarrow\left(T, T_{0}\right)$ and let $T_{1}=\bar{U}, U_{0}=$ interior $T_{0}$; then $f^{-1} T_{1}$ $\subset f^{-1}\left(U_{0}\right)$ and we can find $A$ a closed subpolyhedron of $X$ with $f^{-1} T_{1} \subset A \subset$ $f^{-1}\left(U_{0}\right)$ and hence a regular neighborhood pair $\left(V, V_{0}\right)$ of $A$ with $V \subset f^{-1}\left(U_{0}\right)$. 
If we let $Y=\overline{X-V}$ and $Y_{0}=\overline{X_{0}-V_{0}}$, then the restriction of $f, f_{1}$ maps $\left(Y, Y_{0} \cup \dot{V}\right)$ into $\left(T-T_{1}, T_{0}-T_{1}\right)$, and we claim that, regarded as a map into $\left(T, T_{0}\right), f_{1}$ is cobordant to $f$. Let $F$ be the restriction of $f \cdot \pi_{X}$ and use the cobordism

$(X \times[0,1 / 2] \cup Y \times[1 / 2,1]$,

$$
\begin{array}{r}
X \times 0 \cup X_{0} \times[0,1 / 2] \cup V \times 1 / 2 \cup\left(Y_{0} \cup \dot{V}\right) \times[1 / 2,1] \cup Y \times 1, \\
\left.X_{0} \times[0,1 / 2] \cup V \times 1 / 2 \cup\left(Y_{0} \cup \dot{V}\right) \times[1 / 2,1]\right) .
\end{array}
$$

Note that $f_{1}(Y) \subset T-T_{1}$ and if $f(X) \subset T-U$ then this is a cobordism in $\left(T-U, T_{0}-U\right)$.

This shows $i_{*}$ is onto.

Now let $f:\left(X, X_{0}\right) \rightarrow\left(T-U, T_{0}-U\right)$ such that $i_{*}[f]=0$. By the above note we can assume that $f(X) \subset T-T_{1}$. Let $\left(\left(W, W_{0}, W_{1}\right), F\right)$ be a cobordism of $f$ with 0 in $\left(T, T_{0}\right)$, i.e. $W_{0}=X \cup W_{1}, X \cap W_{1}=\dot{X_{0}}$, and $F:\left(W, W_{1}\right) \rightarrow$ $\left(T, T_{0}\right)$ with $F \mid X=f$. Since $f(X) \subset T-T_{1}, F^{-1}\left(T_{1}\right) \cap X=\varnothing$; so we can first choose $A$ and then a regular neighborhood pair $\left(V, V_{0}\right)$ of $A$ so that

$$
F^{-1}\left(T_{1}\right) \subset V \subset F^{-1}\left(U_{0}\right) \cap(W-X) .
$$

Then the restriction of $F$ to $\left(\overline{W-V}, \overline{W_{0}-V_{0}} \cup \dot{V}, \overline{W_{1}-V_{0}} \cup \dot{V}\right)$ gives a cobordism of $f$ to 0 in $\left(T-U, T_{0}-U\right)$.

EXACTNESS OF THE DIAGRAM $\ldots \Omega_{n}\left(T_{0}\right) \stackrel{i_{*}}{\longrightarrow} \Omega_{n}(T) \stackrel{i_{*}}{\longrightarrow} \Omega_{n}\left(T, T_{0}\right)$ $\stackrel{\partial}{\longrightarrow} \Omega_{n-1}\left(T_{0}\right) \stackrel{i_{*}}{\longrightarrow} \Omega_{n-1}(T)$.

Exactness at $\Omega_{n-1}\left(T_{0}\right):$ If $f:\left(X, X_{0}\right) \rightarrow\left(T, T_{0}\right)$, then $\left(\left(X, X_{0}, \varnothing\right), f\right)$ is a cobordism of $f: X_{0} \rightarrow T$ and 0 . Hence $i_{*} \partial[f]=0$.

If $i_{*}[f]=0$ with $f: X_{0} \rightarrow T_{0}$, then $\left(\left(W, W_{0}, W_{1}\right), F\right)$ maps $W_{1}$ to $\varnothing$ and so $W_{1}=\varnothing$, i.e. $X_{0}=W_{0}$ and $F:\left(W, X_{0}\right) \rightarrow\left(T, T_{0}\right)$ or $\partial[F]=[f]$.

Exactness at $\Omega_{n}\left(T, T_{0}\right):$ If $f: X \rightarrow T$, i.e. $f:(X, \varnothing) \rightarrow(T, \varnothing)$ then $\partial j_{*}[f]$ $=0$ by definition. If $f:\left(X, X_{0}\right) \rightarrow\left(T, T_{0}\right)$ with $\partial[f]=0$ then there exists as in the previous argument, a pair $\left(Y, X_{0}\right) \in \mathcal{F}^{n}$ with $F: Y \rightarrow T_{0}$ extending $f$. By pasting $X^{\prime}=X \cup_{X_{0}} Y \in \mathfrak{F}^{n}$ and $f \cup F: X^{\prime} \rightarrow T$ is cobordant to $f$ in $\left(T, T_{0}\right)$. The cobordism is

$$
\begin{aligned}
& \left(X \times[0,1 / 2] \cup X^{\prime} \times[1 / 2,1],\right. \\
& \left.\quad X \times 0 \cup X_{0} \times[0,1 / 2] \cup Y \times 1 / 2 \cup X^{\prime} \times 1, X_{0} \times[0,1 / 2] \cup Y \times 1 / 2\right)
\end{aligned}
$$

together with the map $f \cup F \cdot \pi_{X^{\prime}}$.

Exactness at $\Omega_{n}(T):$ If $f: X \rightarrow T_{0}$ then $\left((X \times I, X \times I, X \times 1), f \cdot \pi_{X}\right)$ is a cobordism of $f$ to 0 in $\Omega_{n}\left(T, T_{0}\right)$, i.e. $j_{*} \cdot i_{*}=0$. If $j_{*}[f]=0$ where $f: X \rightarrow$ $T$ then there exists $\left(\left(W, W_{0}, W_{1}\right), F\right)$ with $X \subset W_{0}$ and $W_{1} \cap X=\varnothing$, and $F$ extends $f$ mapping $\left(W, W_{1}\right) \rightarrow\left(T, T_{0}\right)$. Hence it can also be regarded as a cobord- 
ism of $\left[F \mid W_{1}\right]+f$ with 0 in $\Omega_{n}(T)$. So $[f]=i_{*}\left[F \mid W_{1}\right]$.

REMARKs. 1. Compactness was assumed to prevent $\Omega_{*}$ from always being 0 ; it is not used in the proof, but for most examples if $f:\left(X, X_{0}\right) \rightarrow\left(T, T_{0}\right)$ then $\left(\left(X \times[0, \infty), X \times 0 \cup X_{0} \times[0, \infty), X_{0} \times[0, \infty)\right), f \cdot \pi\right)$ would be a cobordism to 0 if noncompact pairs were allowed. Proper maps only extend the result.

2. If $\left\{\mathfrak{F}^{n}\right\}$ satisfies Axioms $1-4$, then we can define $\mathfrak{F}_{c}^{n}=\left\{\left(X, X_{0}\right) \in \mathfrak{F}^{n}\right.$ : $X_{0} \subset X$ is collared\}.

One can show that $\left\{\mathfrak{F}_{c}^{n}\right\}$ still satisfies Axioms 1-4.

This is clear for Axioms 1 and 2. For Axiom 3, we first show that for $x \in$ $\dot{V}_{0}, \operatorname{Lk}\left(x ;\left(\dot{V}, \dot{V}_{0}\right)\right) \cong\left(c \operatorname{Lk}\left(x ; \dot{V}_{0}\right), \operatorname{Lk}\left(x ; \dot{V}_{0}\right)\right)$, where $(c X, X)$ for $X$ a compact polyhedron, denotes the cone pair with base $X$ and vertex $c$. By $\left[A_{1}\right.$, p. 424, Corollary 10] it suffices to show that the suspension of the two sides are isomorphic. By Remark 2 after Axioms 1-4, $\left(\dot{V}, \dot{V}_{0}\right)$ is bicollared in $\left(X, X_{0}\right)$. So, $\Sigma \mathrm{Lk}\left(x ;\left(\dot{V}, \dot{V}_{0}\right)\right) \cong \operatorname{Lk}\left(x ;\left(X, X_{0}\right)\right)$. On the other hand, $\Sigma\left(c \operatorname{Lk}\left(x ; \dot{V}_{0}\right), \operatorname{Lk}\left(x ; \dot{V}_{0}\right)\right)$ $\cong\left(c \Sigma \mathrm{Lk}\left(x ; \dot{V}_{0}\right), \Sigma \mathrm{Lk}\left(x ; \dot{V}_{0}\right)\right) \cong\left(\operatorname{Lk}\left(x ; X_{0}\right), \operatorname{Lk}\left(x ; X_{0}\right)\right)$. Now these pairs are isomorphic because $X_{0} \subset X$ is collared. Thus, $\dot{V}_{0} \subset \dot{V}$ is collared (cf. [C, p. 205, Theorem 5.3]). Now because $\left(\dot{V}, \dot{V}_{0}\right) \subset\left(V, V_{0}\right)$ is collared we have, for $x \in V_{0}$ : $\operatorname{Lk}\left(x ;\left(V, V_{0}, \dot{V}, \dot{V}_{0}\right)\right) \cong\left(c_{1} c \operatorname{Lk}\left(x ; \dot{V}_{0}\right), c_{1} \operatorname{Lk}\left(x ; \dot{V}_{0}\right), c \operatorname{Lk}\left(x ; \dot{V}_{0}\right), \operatorname{Lk}\left(x ; \dot{V}_{0}\right)\right)$.

So $\operatorname{Lk}\left(x ;\left(V, \dot{V} \cup V_{0}\right)\right) \cong\left(c^{\prime} \Sigma \operatorname{Lk}\left(x ; V_{0}\right), \Sigma \operatorname{Lk}\left(x ; V_{0}\right)\right)$ because $\left[c_{1}, c\right]=$ $c^{\prime} *\left\{c_{1}, c\right\}$ for $c^{\prime}$ in the interior of $\left[c_{1}, c\right]$. Thus, $\dot{V} \cup V_{0} \subset V$ is collared. Similarly, because $\left(\dot{V}, \dot{V}_{0}\right) \subset\left(Y, Y_{0}\right)$ is collared, $\operatorname{Lk}\left(x ;\left(Y, Y_{0} \cup \dot{V}\right)\right) \cong$ $\left(c \Sigma \mathrm{Lk}\left(x ; \dot{V}_{0}\right), \Sigma \mathrm{Lk}\left(x ; \dot{V}_{0}\right)\right)$ and so $Y_{0} \cup \dot{V} \subset Y$ is collared.

For Axiom 4, regarding the embeddings as inclusions, we have, for $x \in Z_{0}$, that $\operatorname{Lk}\left(x ;\left(X_{1}, Z_{0}\right)\right), \operatorname{Lk}\left(x ;\left(Y_{1}, Z_{0}\right)\right)$ and $\operatorname{Lk}\left(x ;\left(Z, Z_{0}\right)\right)$ are cone pairs as above and we denote the cone vertices by $c_{1}, c_{2}$ and $c_{3}$ respectively. Then:

$$
\begin{aligned}
\operatorname{Lk}\left(x ;\left(X, X_{0}\right)\right) & \cong\left(c_{4} \operatorname{Lk}\left(x ; X_{0}\right), \operatorname{Lk}\left(x ; X_{0}\right)\right) \\
& \cong\left(c_{4} *\left\{c_{1}, c_{3}\right\},\left\{c_{1}, c_{3}\right\}\right) * \operatorname{Lk}\left(x ; Z_{0}\right), \\
\operatorname{Lk}\left(x ;\left(Y, Y_{0}\right)\right) & \cong\left(c_{5} \operatorname{Lk}\left(x ; Y_{0}\right), \operatorname{Lk}\left(x ; Y_{0}\right)\right) \\
& \cong\left(c_{5} *\left\{c_{2}, c_{3}\right\},\left\{c_{2}, c_{3}\right\}\right) * \operatorname{Lk}\left(x ; Z_{0}\right) .
\end{aligned}
$$

When we paste, the links are pasted along $\operatorname{Lk}(x ; Z) \cong c_{3} * \operatorname{Lk}\left(x ; Z_{0}\right)$ :

$$
\begin{aligned}
\operatorname{Lk}\left(x ;\left(X \cup_{Z}\right.\right. & \left.\left.Y, X_{1} \cup Z_{0} Y_{1}\right)\right) \\
& \cong\left(c_{4} *\left\{c_{1}, c_{3}\right\} \cup c_{5} *\left\{c_{2}, c_{3}\right\},\left\{c_{1}, c_{2}\right\}\right) * \operatorname{Lk}\left(x ; Z_{0}\right) .
\end{aligned}
$$

Since $\left(c_{4} *\left\{c_{1}, c_{3}\right\} \cup c_{5} *\left\{c_{2}, c_{3}\right\},\left\{c_{1}, c_{2}\right\}\right) \cong\left(c_{3} *\left\{c_{1}, c_{2}\right\},\left\{c_{1}, c_{2}\right\}\right)$, the link pair is a cone pair and so the result of the pasting is collared.

Furthernore, the bordism groups remain the same. Thus, if $f:\left(X, X_{0}\right) \rightarrow$ 
$\left(T, T_{0}\right)$ with $\left(X, X_{0}\right) \in \mathfrak{F}^{n}$ then by Axioms 1 and $4,\left(\bar{X}, \bar{X}_{0}\right) \in \mathfrak{F}^{n}$ where $\bar{X}=$ $X \cup X_{0} \times[0,1]$ with $X_{0} \subset X$ identified with $X_{0} \times 0$ and $\bar{X}_{0}=X_{0} \times 1 . \bar{X}_{0} \subset$ $\bar{X}$ is collared and if $r:\left(\bar{X}, \bar{X}_{0}\right) \rightarrow\left(X, X_{0}\right)$ is the retraction obtained by flattening the collar, then $F=f \cdot r \cdot \pi_{\bar{X}}$ together with

$$
\begin{aligned}
& \left(\bar{X} \times[0,1 / 2] \cup X \times[1 / 2,1], \bar{X} \times 0 \cup \bar{X}_{0} \times[0,1 / 2] \cup X_{0} \times I \times 1 / 2\right. \\
& \left.\cup X_{0} \times[1 / 2,1] \cup X \times 1, \bar{X}_{0} \times[0,1 / 2] \cup X_{0} \times I \times 1 / 2 \cup X_{0} \times[1 / 2,1]\right)
\end{aligned}
$$

is a cobordism between the maps $f:\left(X, X_{0}\right) \rightarrow\left(T, T_{0}\right)$ and $f \cdot r:\left(\bar{X}, \bar{X}_{0}\right) \rightarrow$ $\left(T, T_{0}\right)$. Similarly, by attaching a collar to the cobordism, we can show that $f$ cobordant to 0 with respect of $\left\{\mathcal{F}^{n}\right\}$ implies $f \cdot r$ cobordant to 0 with respect to $\left\{\mathfrak{F}_{c}^{n}\right\}$.

So we can regard the condition " $X_{0} \subset X$ is collared" as a convenient normalization condition which does not affect the bordism groups.

We say that a bordism sequence $\left\{\mathfrak{F}^{n}\right\}$ has collared boundaries if $\left(X, X_{0}\right) \in$ $\mathcal{F}^{n}$ implies that $X_{0} \subset X$ is collared. We now characterize bordism sequences with collared boundaries in two important special cases.

Definition 6. A locally finite complex $K$ is called totally $n$-dimensional if it satisfies the equivalent conditions: of $K$.

(a) Every simplex of $K$ is a (not necessarily proper) face of some $n$-simplex

(b) $K=$ Closure $\left(I^{n}(|K|)-I^{n-1}(|K|)\right)$.

Since the latter condition is p.l. invariant we can call a locally compact polyhedron $X$ totally $n$-dimensional if the above holds for some (and hence for any) triangulation of $X$.

Proof OF EQUivalence. For any locally finite complex $K$ with $n$-skeleton $K^{n}$, we have:

$$
K^{n}-K^{n-1} \subset I^{n}(K)-I^{n-1}(K) \subset \text { Closure }\left(K^{n}-K^{n-1}\right) .
$$

The left inequality follows from the definition $\left[A_{1}\right.$, pp. 420-421] of intrinsic dimension. For the right, if $\sigma$ is a simplex of $I^{n}(K)-I^{n-1}(K)$, then since the latter is an open $n$-manifold, $\sigma$ is the face of some $n$-simplex. It follows that the closures of $K^{n}-K^{n-1}$ and $I^{n}(K)-I^{n-1}(K)$ are the same.

REMARK. Let $X$ be totally $n$-dimensional. If $X_{0} \subset X$ is collared, then $X_{0}$ is totally ( $n-1)$-dimensional. If $V$ is a subpolyhedron of $X$ and is the closure of its interior, then $V$ is totally $n$-dimensional. Putting these two results together, if $x \in X$, then $\operatorname{Lk}(x ; X)$ is totally $(n-1)$-dimensional.

DEFINITION 7. Let $\left\{\mathcal{F}^{n}\right\}$ be a bordism sequence with collared boundaries. Call $\left\{\mathfrak{F}^{n}\right\}$ ungraded if $\mathfrak{F}^{n}=\mathfrak{F}^{m}$ for all $m$ and $n$. Call $\left\{\mathfrak{F}^{n}\right\}$ dimension graded if 
$\left(X, X_{0}\right) \in \mathcal{F}^{n}$ implies $X=\varnothing$ or $X$ is totally $n$-dimensional.

REMARK. In either case, if we let $\mathfrak{F}=\bigcup_{n} \mathfrak{F}^{n}$ then $\mathfrak{F}$ characterizes $\left\{\mathfrak{F}^{n}\right\}$, that is:

Ungraded case. $\left(X, X_{0}\right) \in \mathcal{F}^{n}$ iff $\left(X, X_{0}\right) \in \mathcal{F}$.

Dimension graded case. $\left(X, X_{0}\right) \in \mathcal{F}^{n}$ iff $\left(X, X_{0}\right) \in \mathfrak{F}$ and $X=\varnothing$ or $X$ is totally $n$-dimensional.

Definition 8. A class $\mathfrak{D}$ of compact polyhedra is called a class of singularities if it satisfies:

(a) $\varnothing \in \mathcal{D}$.

(b) $X \in \mathfrak{D}$ and $X \cong Y$ implies $Y \in \mathfrak{D}$.

(c) $X \in \mathfrak{D}$ iff $\Sigma X \in \mathfrak{D}$.

Since the suspensions of $\varnothing$ are spheres, $\mathfrak{D}$ includes all spheres.

For $\mathcal{D}$ a class of singularities define:

$\mathfrak{F}_{0}=\left\{\left(X, X_{0}\right):\left(X, X_{0}\right)\right.$ is a compact pair with $X_{0} \subset X$ collared and $x \in X-X_{0}$ implies $\left.\operatorname{Lk}(x ; X) \in \mathbb{D}\right\}$.

$\mathfrak{F}_{0}^{n}=\left\{\left(X, X_{0}\right) \in \mathfrak{F}_{\mathfrak{D}}\right.$ with $\left(X, X_{0}\right)$ totally $n$-dimensional or $\left.X=\varnothing\right\}$.

PROPOSITION 8. Let $\mathfrak{D}$ be a class of singularities, then $\mathfrak{F}_{\mathfrak{D}}$ is an ungraded bordism sequence and $\left\{\mathcal{F}_{פ}^{n}\right\}$ is a dimension graded bordism sequence.

Proof. The preservation of collaring by the operations of the axioms is proved as in Remark 2 following Proposition 7. In the dimension graded case, the preservation of total $n$-dimensionality is easy (cf. the Remark following Definition 6). The links remain in $\mathcal{D}$ because if $X_{0} \subset X$ is bicollared and $x \in X_{0}$, then $\mathrm{Lk}(x ; X) \cong \Sigma \mathrm{Lk}\left(x ; X_{0}\right)$ and so $\operatorname{Lk}(x ; X) \in \mathcal{D}$ iff $\operatorname{Lk}\left(x ; X_{0}\right) \in \mathcal{D}$. The details are left to the reader.

DEFINITION 9. Let $\left\{\mathfrak{F}^{n}\right\}$ be a bordism sequence and let $\mathfrak{F}=\bigcup_{n} \mathfrak{F}^{n}$. We define the singularity class $\mathcal{D}_{\mathfrak{Z}}$ by the following equivalent equations:

$$
\begin{aligned}
\mathscr{D}_{\mathfrak{g}} & =\{\text { compact polyhedra } X: \Sigma X \in \mathcal{F}\} \cup\{\varnothing\} \\
& =\{X: \exists Y \in \mathcal{F}, y \in Y \text { such that } \operatorname{Lk}(y ; Y) \cong X\} \cup\{\varnothing\} \\
& =\{\text { compact polyhedra } X:(c X, X) \in \mathcal{F}\} \cup\{\varnothing\} .
\end{aligned}
$$

Proof of EQuivalence. Since $\operatorname{Lk}(v ; \Sigma X) \cong X$ for $v$ a suspension vertex, the first set is contained in the second. On the other hand, if $Y \in \mathcal{F}$, then the regular neighborhood and boundary of $y$ in $Y$ is isomorphic to $(c \operatorname{Lk}(y ; Y), \operatorname{Lk}(y ; \eta))$ and so the second set is contained in the third by Axioms 1 and 3. Finally, $\Sigma X \cong c X \cup c^{\prime} X$, union along $X$, and so the third set is contained in the first, by Axiom 4. 
REMARK. If $\mathfrak{F} \neq\{\varnothing\}$ then $\mathfrak{D}_{\mathfrak{F}}$ is a singularity class. For if $X \in \mathfrak{D}_{\mathfrak{F}}$ and $X \neq \varnothing$ then $\operatorname{Lk}((y, 1 / 2), Y \times I) \cong \Sigma \mathrm{Lk}(y, Y)$ implies that $\Sigma X \in \mathcal{D}_{\mathfrak{z}}$ by Axiom 2 . If $\Sigma X \in \mathfrak{D}_{\mathfrak{F}}$, then $(c \Sigma X, \Sigma X) \in \mathfrak{F}$ and so $\Sigma X \in \mathcal{F}$ by Axiom 2 and so $X \in \mathfrak{D}_{\mathfrak{F}}$. Finally, $\mathfrak{f}$ contains some nonempty polyhedron and so $\mathfrak{D}_{\mathfrak{F}}$ contains some sphere. By suspending and desuspending it contains all spheres. Thus, $\mathfrak{D}_{\mathfrak{F}}$ satisfies (a), (b) and (c) above.

Proposition 9. (a) Let $\left\{\mathcal{F}^{n}\right\}$ be an ungraded bordism sequence and let $\mathfrak{D}=\mathfrak{D}_{\mathfrak{F}}$. Then $\mathfrak{F}_{\mathfrak{Q}}=\mathfrak{F}$.

(b) Let $\left\{\mathfrak{F}^{n}\right\}$ be a dimension graded bordism sequence and let $\mathfrak{D}=\mathfrak{D}_{\mathfrak{f}}$. Then $\mathfrak{f}_{\mathbb{Q}}^{n}=\mathfrak{F}^{n}$ for all $n$.

Proof. It suffices to show that $X \in \mathfrak{F}_{\mathfrak{S}}$ implies $X \in \mathfrak{F}$ in (a), and $X \in \mathfrak{F}_{\mathfrak{S}}^{n}$ implies $X \in \mathcal{F}^{n}$ in (b). The result for pairs then follows because if $\left(X, X_{0}\right) \in \mathfrak{F}_{\Im}^{n}$ then the double consisting of two copies of $X$ pasted along $X_{0}$ is in $\mathcal{F}_{\mathcal{Q}}^{n}$ and hence is in $\mathcal{F}^{n}$. But since $X_{0} \subset X$ is collared, the pair $\left(X, X_{0}\right)$ is isomorphic to the regular neighborhood and boundary of $X$ in the double. So $\left(X, X_{0}\right) \in \mathfrak{F}^{n}$ by Axioms 1 and 3. The result for pairs is obtained similarly in case (a).

We prove the result in the dimension graded case. The proof is easier in the ungraded case as we can just forget about the grading, but otherwise it is the same.

Let $K$ be a finite complex with $|K| \in \mathfrak{F}_{\mathbb{Q}}^{n}$ and let $K_{1} \subset K_{2} \subset \ldots \subset K_{N}=$ $K$ be a sequence of subcomplexes so that $K_{1}$ is a vertex of $K$ and $K_{i+1}=K_{i} U$ $\sigma_{i+1}$. We show by induction that if $V_{i}$ is a regular neighborhood of $K_{i}$ in $K$, then $\left(V_{i}, \dot{V}_{i}\right) \in \mathfrak{F}^{n}$. For $i=N$ this says that $|K| \in \mathfrak{F}^{n}$. Note that by the remark after Definition 6 , the $V_{i}$ 's are totally $n$-dimensional. By uniqueness of regular neighborhoods and Axiom 1, either all of the regular neighborhoods of $K_{i}$ lie in $\mathfrak{f}^{n}$ or none of them do.

Initial step. For any point $x \in K$, if $V$ is a regular neighborhood of $x$, then $(V, \dot{V}) \cong(c \operatorname{Lk}(x ;|K|), \operatorname{Lk}(x ;|K|)) . \operatorname{Lk}(x ;|K|) \in \mathcal{D}$ because $K \in \mathcal{J}_{\mathfrak{Q}}^{n}$ and so the pair is in $\mathcal{f}^{n}$ by Definition 9 . This applies, in particular to the vertex in $K_{1}$.

Inductive step. We will show that a regular neighborhood $V_{i+1}$ of $K_{i+1}$ can be obtained as a union of $V_{i}$ and $U_{i+1}$, where $V_{i}$ is a regular neighborhood of $K_{i}, U_{i+1}$ is a regular neighborhood of the barycentre of $\sigma_{i+1}$, and $V_{i} \cap U_{i+1}$ $=\dot{V}_{i} \cap \dot{U}_{i+1}$ is a regular neighborhood of something both in $\dot{V}_{i}$ and $\dot{U}_{i+1}$. Then, $V_{i}$ is in $\mathcal{f}^{n}$ by induction hypothesis, $U_{i+1}$ is in $\mathfrak{f}^{n}$ by the initial step and so $\left(V_{i+1}, \dot{V}_{i+1}\right) \in \mathcal{F}^{n}$ by Axioms 3 and 4 .

To prove the regular neighborhood result we have to do a little work with Cohen's parametrization lemma [C, p. 194, Lemma 2.14]. Let $K^{\prime}$ be the barycentric subdivision of $K$ and let $p: K^{\prime} \rightarrow[a b c]$ be a simplicial map to a two simplex with $p$ mapping $K_{i}^{\prime}$ to $a$, the barycentre of $\sigma_{i+1}$ to $b$ and the remaining 
vertices of $K^{\prime}$ to $c$. We use two derived subdivisions of [abc] so that $\pi: \eta[a b c]$ $\rightarrow \eta[a b]$ and $\bar{\pi}: \bar{\eta}[a b c] \rightarrow \eta[a b]$ are simplicial, where $\pi$ and $\bar{\pi}$ are the simplicial retractions of $[a b c]$ onto $[a b]$ with $\pi(c)=b$ and $\bar{\pi}(c)=a$. We obtain corresponding derived subdivisions $\eta K^{\prime}$ and $\bar{\eta} K^{\prime}$ so that $p: \eta K^{\prime} \rightarrow \eta[a b c]$ and $p: \bar{\eta} K^{\prime} \rightarrow$ $\bar{\eta}[a b c]$ are still simplicial. See the figure below:
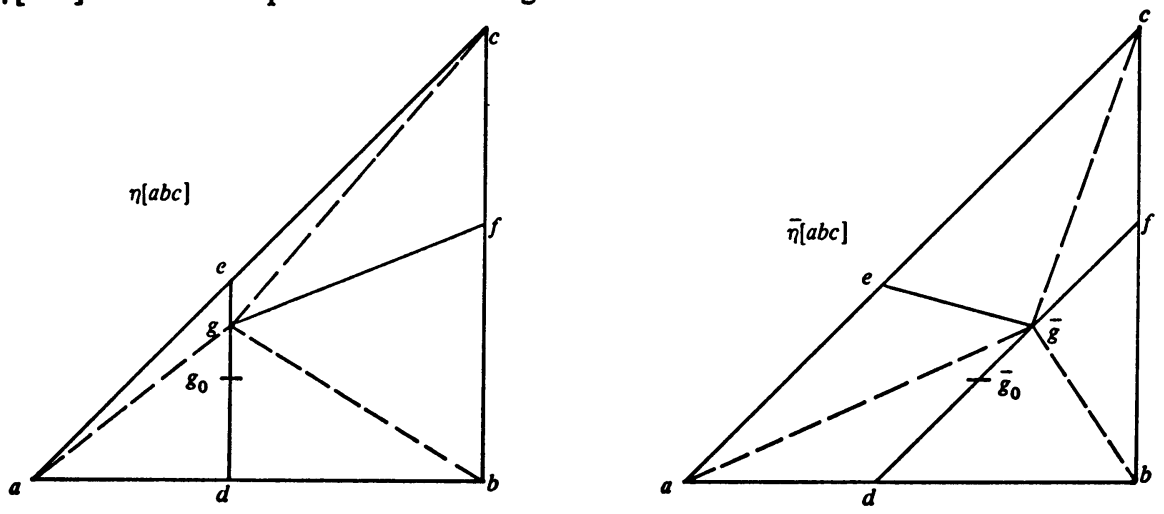

Applying the parametrization lemma to the diagram for $\eta[a b c]$, we get:

$$
\begin{aligned}
& \left(p^{-1}(a d g e), p^{-1}(d g e)\right)=\left(V_{i}, \dot{V}_{i}\right), \quad\left(p^{-1}(b d g f), p^{-1}(d g f)\right)=\left(U_{i+1}, \dot{U}_{i+1}\right), \\
& p^{-1}(d g)=V_{l} \cap U_{i+1}=\dot{V}_{i} \cap \dot{U}_{i+1} .
\end{aligned}
$$

Furthermore, $p^{-1}\left(d g_{0}\right)$ is a regular neighborhood of $p^{-1}(d)$ in $V_{i}$ with boundary $p^{-1}\left(g_{0}\right)$. If we choose $g_{0}$ as the vertex for $[a b c]$ instead of $g$ and correspondingly change the choices of vertices for those simplices of $K^{\prime}$ which map onto $[a b c]$, then we obtain different derived subdivisions $\eta_{0} K$ and $\eta_{0}[a b c]$. Of course, $\eta_{0} K^{\prime}$ is simplicially isomorphic to $\eta K^{\prime}$ and because $e, g, g_{0}, d$ are colinear,this isomorphism takes $\left(p^{-1}\left(d g_{0} e\right), p^{-1}\left(d g_{0}\right), p^{-1}\left(g_{0}\right)\right)$ onto $\left(p^{-1}(d g e), p^{-1}(d g)\right.$, $\left.p^{-1}(g)\right)$ and is the identity on $p^{-1}(d)$. Hence, $p^{-1}(d g)$ is a regular neighborhood with boundary $p^{-1}(g)$ of $p^{-1}(d)$ in $p^{-1}(d g e)$, i.e. $V_{i} \cap U_{i+1}$ is a regular neighborhood in $\dot{V}_{i}$. Next, use the simplicial isomorphism of $\eta K^{\prime}$ with $\bar{\eta} K^{\prime}$ and a similar sliding argument on the colinear points $d, \bar{g}_{0}, \bar{g}, f$ to get that $p^{-1}(d g)$ is a regular neighborhood of $p^{-1}(d)$ in $p^{-1}(d g f)$, i.e. $V_{i} \cap U_{i+1}$ is a regular neighborhood of $p^{-1}(d)$ in $\dot{U}_{i+1}$.

Remarks. 1. This result is really a negative one. It shows that all of the examples are of a particular type. The culprit is the pasting axiom. To get more general bordism theories, one must introduce additional structure, e.g. orientation, on the pairs and only allowing pastings which preserve the additional structure, e.g. opposite orientations on $Z$. In these cases, the structures induced on $X \times 0$ and $X \times 1$ from $X \times I$ are often different so that one does not obtain a $Z_{2}$ theory any more.

2. If $X \in \mathfrak{D}$ implies $X$ is totally $n$-dimensional for $n=\operatorname{dim} X$, then the 
ungraded bordism group $\Omega\left(T, T_{0}\right)$, associated to the ungraded bordism family $\mathfrak{F}_{\mathfrak{D}}$ is just the direct sum $\Sigma_{n} \Omega_{n}\left(T, T_{0}\right)$ where $\Omega_{*}\left(T, T_{0}\right)$ are the bordism group associated to the dimension graded family $\left\{\mathcal{Z}_{\Xi}^{n}\right\}$. This is left to the reader with the hint that if $X$ is a polyhedron such that the $\operatorname{Lk}(x ; X)$ is totally $n_{x}$-dimensional for each $x \in X$ (with $n_{x}$ varying with $x$ ), then each component of $X$ is totally $n$-dimensional (with $n$ varying with the choice of component). Hence, any element of $\mathfrak{F}_{\mathfrak{g}}$ is a disjoint union of elements of $\bigcup_{n} \mathfrak{F}_{פ}^{n}$.

Note that if we besin with a dimension graded family $\left\{\mathfrak{F}^{n}\right\}$ then the corresponding family $\mathfrak{D}_{\mathfrak{F}}$ satisfies the above condition.

In general, however, if $\mathcal{D}$ does not satisfy this condition then the map $\Sigma_{n} \Omega_{n}$ $\rightarrow \Omega$ induced by the inclusion of $\bigcup_{n} \mathfrak{F}_{9}^{n}$ in $\mathfrak{F}_{\mathfrak{S}}$, need not be injective. For example, look at the Euler space bordism groups of the next section.

Euler space cobordism. We will now turn to an example of a $Z_{2}$ bordism theory which comes from Euler spaces.

Let $\mathfrak{F}^{E}=\left\{\left(X, X_{0}\right)\right.$ is a compact Euler pair $\}$. If we let $\mathfrak{F}^{n}=\mathfrak{F}^{E}$ for all $n \in Z$ we obtain a sequence satisfying Axioms $1,2,3,4$ and hence an ungraded bordism theory $\Omega^{E}$ with exact triangle for topological pair $\left(T, T_{0}\right)$ :

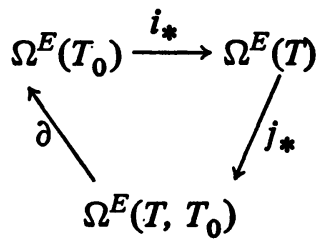

PROPOSITION 10. There is a natural homomorphism

$$
\text { S: } \Omega^{\dot{E}}\left(T, T_{0}\right) \rightarrow H_{*}\left(T, T_{0}\right) \quad \text { (total } Z_{2} \text { homology) }
$$

defined by $S[f]=\Sigma_{i} f_{*} w_{i}\left(X, X_{0}\right)$ where $f:\left(X, X_{0}\right) \rightarrow\left(T, T_{0}\right)$, i.e. $S$ maps $[f]$ to the image under $f_{*}$ of the total Stiefel-Whitney homology class.

Proof. $S$ is well defined by Proposition 5. It is clearly a natural homomorphism commuting with boundaries.

PROPOSITION 11. (a) $X$ and $Y \in \mathcal{F}$ are cobordant if and only if $\chi(X) \equiv$ $\chi(Y)$.

(b) For compact p.l. pairs $\left(T, T_{0}\right), S$ is an isomorphism.

Proof. (1) $S: \Omega^{E}(p) \rightarrow H_{*}(p)=Z_{2}$ ( $p$ a point) is onto: Let $f: X \rightarrow p$ be the identity map on $p . w_{0}(p) \neq 0$.

(2) If $\chi(X) \equiv \chi(Y)$ then the pair $\left(c X \cup_{c} c Y, X \cup Y\right)$ is a cobordism between $X$ and $Y$, since $\chi(X \cup Y) \equiv 0$.

(3) $S: \Omega^{E}(p) \rightarrow H_{*}(p)=Z_{2}$ is iso: By (1) it is onto and by (2) there is at most one nonzero class in $\Omega^{E}(p)$. 
(4) If there existed $X$ cobordant to $Y$ with $\chi(X) \neq \equiv(Y)$ then, by (2), $\Omega^{E} p$ $=0$ which is impossible by (3). This proves (a).

(5) $S: \Omega^{E}\left(E^{n}, S^{n-1}\right) \rightarrow H_{*}\left(E^{n}, S^{n-1}\right)$ is iso by induction on $n$ using naturality of $S$ with respect to the Mayer-Vietoris boundary, cf. Proposition 4.

(6) $S: \Omega^{E}(K) \rightarrow H_{*}(K)$ is iso for any finite complex $K$ by induction on the number of cells. $K=K_{0} \cup \sigma$ with $\sigma \cap K_{0}=\partial \sigma$. Then by inductive hypothesis $S$ is iso on $K_{0}$ and by excision and (5) it is iso on $\left(K, K_{0}\right)$ and so $S$ is iso on $K$ by the five lemma.

(7) $S: \Omega^{E}\left(K, K_{0}\right) \rightarrow H_{*}\left(K, K_{0}\right)$ is iso for any finite complex pair $\left(K, K_{0}\right)$ by the five lemma and (6) for $K$ and $K_{0}$ separately.

Corollary 1. If $\left(X, X_{0}\right)$ is a compact Euler pair, then $\chi\left(X_{0}\right) \equiv 0$.

Another corollary is the converse of Proposition 5.

Corollary 2. If $f:\left(X, X_{0}\right) \rightarrow\left(T, T_{0}\right)$ and $g\left(Y, Y_{0}\right) \rightarrow\left(T, T_{0}\right)$ are maps of compact Euler pairs to compact p.l. pairs, then if $f_{*} w_{i}\left(X, X_{0}\right)=g_{*} w_{i}\left(Y, Y_{0}\right)$ for all $i$, then $f$ and $g$ are cobordant.

George Cooke showed me how to contrast this with manifold cobordism. $f$ and $g$ are cobordant iff the generalized Stiefel-Whitney numbers are equal (see [C-F; p. 47, Theorem 17.2]), i.e. iff

$$
\left\langle f^{*} z \cup w^{i_{1}} \cup \ldots \cup w^{i_{k}}, \mu_{X}\right\rangle=\left\langle g^{*} z \cup w^{i_{1}} \cup \ldots \cup w^{i_{k}}, \mu_{Y}\right\rangle
$$

where $\mu_{X}$ and $\mu_{Y}$ are the fundamental homology classes, $U$ is cup product and $z$ is an arbitrary cohomology class of $\left(T, T_{0}\right)$ of codimension $i_{1}+\ldots+i_{k}$. The $w^{i}$ 's are the usual Stiefel-Whitney cohomology classes, which by Cheeger, Halperin, Toledo etc. are dual to the $w_{i}$ 's. Since $\cap=$ intersection is dual to cup product, we have

$$
\begin{aligned}
\left\langle f^{*} z \cup w^{i_{1}} \cup \ldots \cup w^{i_{k}}, \mu_{X}\right\rangle & =\left\langle f^{*} z, w_{i_{1}} \cap \ldots \cap w_{i_{j}}\right\rangle \\
& =\left\langle z, f_{*}\left(w_{i_{1}} \cap \ldots \cap w_{i_{k}}\right)\right\rangle .
\end{aligned}
$$

Thus, $f$ and $g$ are Euler space cobordant iff $f_{*} w_{i}=g_{*} w_{i}$ for all $i$. They are manifold cobordant iff $f_{*}\left(w_{i_{1}} \cap \ldots \cap w_{i_{k}}\right)=g_{*}\left(w_{i_{1}} \cap \ldots \cap w_{i_{k}}\right)$. In the first case the Stiefel-Whitney homology classes are related and in the second the generated intersection algebra classes are all related.

Finally, using the fact that ordinary cobordism maps onto homology by evaluation on the fundamental class, we can find a cobordism between any Euler space and a disjoint union of manifolds.

Proposition 12. (a) If $z \in H_{n}(T)$ then there exists $X$ a disjoint union of compact manifolds of dimension $\leqslant n$ and a map $f: X \rightarrow T$ such that $z=S[f]$. 
(b) If $X$ is a compact Euler space then there exists $Y$ a disjoint union of manifolds of dimension $\leqslant$ dimension of $X$ and a map $r: Y \rightarrow X$ such that $r$ is cobordant to $1_{X}$.

Proof. (a) By induction on $n$. For $n=0$ it is obvious. There exists a manifold $X_{0}$ of dimension $n$ and a map $f_{0}: X_{0} \rightarrow T$ such that $f_{0 *} \mu_{X_{\sigma}}=$ $f_{0} * w_{n}\left(X_{0}\right)=z$. So $z+S\left[f_{0}\right]=\Sigma_{i<n} f_{0} * w_{i}\left(X_{0}\right)$. By induction, there exists $X_{1}$ a disjoint of manifolds and $f_{1}: X_{1} \rightarrow T$ such that $S\left[f_{1}\right]=z+S\left[f_{0}\right]$. Let $X=$ $X_{0} \cup X_{1}$ and $f=f_{0} \cup f_{1}$.

(b) Apply (a) to the classes of $S\left[1_{X}\right]$, for the existence of $r: Y \rightarrow X$. The cobordism follows from Corollary 2 of Proposition 9. Note that $\operatorname{dim} Y \leqslant$ $\max \left\{i: w_{i}(X) \neq 0\right\}$.

Finally, we contrast the ungraded theory we have been looking at with the dimension graded bordism theory obtained from the class $\mathfrak{D}$ of Euler spaces with even Euler characteristic via Proposition 8. Note that if $X_{1}$ and $X_{2}$ are totally $n$-dimensional Euler spaces with the same Euler characteristic mod 2, then the cobordism $c X_{1} \cup c X_{2}$ is totally $(n+1)$-dimensional. Hence, by Corollary 1, we can compute the coefficient groups: $\Omega_{n}$ (point) $=Z_{2}$ for all $n \geqslant 0$. This is suggestive of $K$-theory. At any rate notice that $\Omega \neq \Sigma_{n} \Omega_{n}$.

NOTES ADDED IN PROOF. 1. 12(b) extends to pairs $\left(X, X_{0}\right)$ by removing a neighborhood of $r^{-1}(c)$ from $r: Y \rightarrow X \cup c X_{0}$.

2. Sullivan and the referee showed me that $12(\mathrm{~b})$ and the product formula for S-W classes on manifolds yield the corresponding result for Euler spaces.

\section{BIBLIOGRAPHY}

[A ] E. Akin, Manifold phenomena in the theory of polyhedra, Trans. Amer. Math. Soc. 143 (1969), 413-473. MR 40 \#6544.

[A2] Transverse cellular mappings of polyhedra, Trans. Amer. Math. Soc. 169 (1972), 401-438.

[C] M. Cohen, A general theory of relative regular neighborhoods, Trans. Amer. Math. Soc. 136 (1969), 189-229. MR 40 \#2052.

[C-F] P. Conner and E. E. Floyd, Differentiable periodic maps, Ergebnisse der Mathematik und ihrer Grenzgebiete, N. F., Bd. 33, Academic Press, New York; Springer-Verlag, Berlin, 1964. MR 31 \#750.

[Ch] G. Cheeger, A combinatorial formula for Steifel-Whitney classes Topology of Manifolds (J. C. Cantrell and C. H. Edwards Editors), Markham, Chicago, Ill., 1971.

[G] L. C. Glaser, Geometrical combinatorial topology, Vol. 1, Van Nostrand Reinhold, Princeton, N. J., 1970.

[H-T] S. Halperin and D. Toledo, Stiefel-Whitney homology classes, Ann. of Math. (2) 96 (1972), 511-525. MR 47 \#1072.

[S] D. Sullivan, Combinatorial invariants of analytic spaces, Proc. Liverpool Singularities-Sy mposium I, Lecture Notes in Math, no. 192, Springer-Verlag, New York, 1971.

DEPARTMENT OF MATHEMATICS, CITY COLLEGE (CUNY), NEW YORK, NEW YORK 10031 